\title{
Incidence of Lymphatic Metastasis to Neck Nodes Level Ilb in Neck Dissection for Head and Neck Cancers: A Retrospective Study
}

\author{
${ }^{1}$ Faisal Al Zahrani, ${ }^{2}$ Khalid Al-Qahtani, ${ }^{3}$ Mohammed Alshahrani, ${ }^{4}$ Khamis Almufargi, ${ }^{5}$ Abdullah Alkhudhayri \\ ${ }^{6}$ Labeb Obad, ${ }^{7}$ Tahera Islam
}

\begin{abstract}
Introduction: Selective neck dissection (SND) is performed to prevent head and neck cancers metastasis. We tried to determine the incidence of level Ilb lymph nodes metastasis and it is associations in head and neck cancers for selection of patients requiring SND.
\end{abstract}

Materials and methods: A retrospective study was conducted on 57 patients who underwent surgical removal of the head and neck tumor by 84 neck dissections. Fisher exact test was used to measure the association between positive Ilb nodes and the other variables.

Results: Nine (15.8\%) of 57 patients showed level Ilb lymph nodes metastasis comprising $10.71 \%$ of the 84 neck dissections. Six $(66.66 \%)$ were associated with oral cavity cancers, $8(88.9 \%)$ with squamous cell carcinoma (SCC), $6(66.66 \%)$ with T4 tumor. Five $(55.6 \%)$ were $\mathrm{N} 2 \mathrm{~b}$, and $7(77.8 \%)$ were found in $\mathrm{N}+$ necks. All (100\%) positive Ilb nodes were associated with metastatic level Ila. Significant associations were found between positive Ilb nodes and N2b $(p=0.005)$, clinically $N+$ necks $(p=0.005)$ and IIa $(p<0.01)$.

Conclusion: The incidence of level Ilb nodes metastasis is high so they should be removed in any oral tumor, SCC, advanced staging, $\mathrm{N}+$ necks or positive lla lymph nodes metastasis.

Keywords: Head neck cancer, Metastasis, Neck lymph node, Level Ilb lymph node.

How to cite this article: Al Zahrani F, Al-Qahtani K, Alshahrani M, Almufargi K, Alkhudhayri A, Obad L, Islam T. Incidence of Lymphatic Metastasis to Neck Nodes Level Ilb in Neck Dissection for Head and Neck Cancers: A Retrospective Study. Int J Head Neck Surg 2014;5(3):99-103.

Source of support: Nil

Conflict of interest: None

\section{INTRODUCTION}

Head and neck cancers include cancers of the lips, mouth, nasal cavity, paranasal sinuses, pharynx and larynx. Most of these cancers are squamous cell carcinomas (SCCs), and

\footnotetext{
1,3-7 Resident, ${ }^{2}$ Consultant

${ }^{1-7}$ Department of Otolaryngology, Head and Neck Surgery King Saud University, Saudi Arabia
}

Corresponding Author: Khalid Al-Qahtani, Consultant Department of Otolaryngology, Head and Neck Surgery, King Saud University, Saudi Arabia, Phone: 00966114774136 e-mail: kqresearch@hotmail.com they usually metastasized locally to the cervical lymph nodes. ${ }^{1,2}$ In 1991, the American Academy of Otolaryngology, Head and Neck Surgery (AAO-HNS) sponsored the Committee for Head and Neck Surgery and Oncology and started to develop a classification system for neck dissections to systematize the terminologies used to describe head and neck operations. In 2002, the classification was modified and is currently endorsed by both the AAO-HNS and the American Society for Head and Neck Surgery (ASHNS). According to this classification, lymph nodes location in the neck divided to six levels. ${ }^{3}$

Level II specifically extends from the skull base, at the lower level of the bony margin of the jugular fossa, to the level of the lower border of the hyoid bone. ${ }^{3}$ It lies anterior to the posterior border of the sternocleidomastoid muscle (SCM) and posterior to a vertical line drawn from the posterior edge of the submandibular gland. ${ }^{3}$ Level II is further subdivided into IIa and IIb. The spinal accessory nerve (SAN), which travels obliquely across this area, is used as a landmark to subdivide this group of lymph nodes to IIa, the part that lies anteriorly, and IIb often called 'submuscular recess', the part that lies posterior to SAN. ${ }^{3,4}$ Cancers that arise from the oral cavity, nasal cavity, nasopharynx, oropharynx, hypopharynx, larynx, and parotid gland have a high risk to metastasize to level II lymph nodes; however, oropharyngeal cancers have a higher chance for metastases to level IIb lymph nodes than others. ${ }^{3}$

Selective neck dissection (SND) is performed to prevent head and neck cancers metastasis. Dissection of the upper jugular vein or SAN in the posterior region of level II is difficult technique in SND. ${ }^{4}$ On the other hand, it is worrisome when the surgeon preserves them. Some authors strongly recommend dissection for all patients with positive cervical lymph node tumors and those affecting the parotid gland, skin, tongue and scalp tumors. ${ }^{5,6}$ However, in clinically negative neck lymph nodes, many authors do not advocate SND. ${ }^{4,7,8}$ One reason for that is, the incidence of metastases to level IIb lymph nodes is low; therefore, dissection of this level may be unnecessary. ${ }^{8}$ Furthermore, additional operating time may be required since the operation of this area involves 
many delicate structures as SAN, which lead to increase morbidity like shoulder dysfunction postoperatively. ${ }^{9}$

There is scarcity of literatures on SND and incidence of level IIb lymph nodes metastatic head and neck tumors in Saudi Arabia. We conducted this study to determine the prevalence of level IIb lymph nodes metastasis among patients who had head and neck cancers in King Fahd Medical City, Riyadh, Saudi Arabia.

\section{MATERIALS AND METHODS}

Fifty-seven patients who had primary not metastasized head and neck cancers underwent surgical procedure to the primary site of the tumor with neck dissection at the Department of Otorhinolaryngology, Head and Neck Surgery, in King Fahad Medical City (KFMC) from March 2007 to January 2010. These patients were analyzed retrospectively in KFMC from July 2011 to November 2012. Research was approved by the institutional review board of KFMC, Ministry of Health, Riyadh, Saudi Arabia and the registration number.

Exclusion criteria were any previous head and neck tumor that was treated surgically, by radiotherapy and/or chemotherapy. Thyroid tumors, proven distant metastasis and positive clinical neck lymph nodes $(\mathrm{N}+)$ of unknown primary site of the tumor were all excluded. Also patients with recurrent head and neck cancers were excluded from the study. Any patient with head and neck tumor who had undergone neck dissection but did not have level IIb lymph nodes removed, labeled and processed separately was excluded from the study.

Fifty-seven patients were enrolled in this study, 32 were males and 25 were females (Table 1). Each side of the neck was considered separately in patients with bilateral neck dissection. High risk patients depending on pathological TNM staging were treated with postoperative radiation therapy. Guidelines of the American Joint Committee on Cancer (2007) were used to stage the cancer. Clinical staging of neck lymph nodes metastasis was based on physical examination and preoperative CT scan.

Statistical analysis to find out the associations between positive level IIb lymph node and other factors (level IIa lymph nodes metastasis, other positive neck lymph nodes, age, gender, primary site and type of the tumor) were calculated by cross-tabulation using Fisher exact test. $\mathrm{p}<0.05$ was considered statistically significant.

\section{RESULTS}

Fifty-seven patients between 16 and 80 years with a mean of $55.9 \pm 16.7$ participated in our study. A total of 84 neck dissections were performed on them, 27 patients underwent bilateral neck dissections and 30 unilateral. Among them, 72 (85.7\%) were SND, 9 (10.7\%) were modified radical neck dissections and $3(3.6 \%)$ were radical neck dissections. Level IIb lymph nodes involvement by metastasis were in 9 (15.8\%) out of 57 patients, representing $10.71 \%$ out of the total 84 neck dissections. Three of them were males and 6 were females with no significant associations noticed in respect to age or gender.

\section{Site of the Tumor}

Most of the tumors were located in the oral cavity, 43 (75.4\%) followed by the larynx 7 (12.3\%) (Table 1). Most of the oral tumors 12 were excised from the tongue followed by 9 patients from the lower alveolar area, then from buccal mucosa in 7 patients. The remaining were 6 from the maxilla, 3 from the floor of the mouth, 2 from the upper alveolus, 2 from the lower lip, and 1 each from the hard palate and the submandibular gland. In relation to the primary site of the tumor, $6(66.66 \%)$ of the nine positive level IIb lymph nodes were associated with the oral cavity cancers $(p=0.386)$, 2 with the larynx ( $p=0.304)$ and 1 with the hypopharynx ( $p=0.409)$. On the other way, $6(13.95 \%)$ of the total 43 oral cavity cancers developed level IIb lymph nodes metastasis.

\section{Type of the Tumor}

All patients were histopathologically diagnosed to have head and neck malignant tumors, $46(80.7 \%)$ patients with SCC, 3 (5.3\%) with adenocarcinoma, 3 (5.3\%) with adenoid cystic carcinoma and the remaining five (8.77\%) were malignant melanoma, mucoepidermoid carcinoma, osteosarcoma, pleomorphic sarcoma and

Table 1: Clinical data of study patients $(\mathrm{n}=57$, positive level Ilb $=9$ )

\begin{tabular}{lll}
\hline Sites of the primary tumor & Patients (\%) & Positive level IIb (\%) \\
\hline Oral cavity & $43(75.4)$ & 6 \\
Oropharynx & $2(3.5)$ & 0 \\
Hypopharynx & $1(1.8)$ & 1 \\
Larynx & $7(12.3)$ & 2 \\
Parotid gland & $3(5.3)$ & 0 \\
Lacrimal gland & $1(1.8)$ & 0 \\
Staging of the tumor & & \\
T1 & $9(15.8)$ & 0 \\
T2 & $13(22.8)$ & 1 \\
T3 & $7(12.3)$ & 2 \\
T4 & $28(49.1)$ & 6 \\
N0 & $30(52.6)$ & 0 \\
N1 & $13(22.8)$ & 2 \\
N2a & $3(5.3)$ & 2 \\
N2b & $10(17.5)$ & 5 \\
N2c & $1(1.8)$ & 0 \\
\hline
\end{tabular}


rhabdomyosarcoma. Eight (88.9\%) out of the nine level IIb lymph nodes metastasis were associated with SCC with no statistical significant association $(\mathrm{p}=0.442)$, and the remaining one (11.1\%) positive level IIb lymph node was associated with adenoid cystic carcinoma. On the other hand, these 8 positive level IIb nodes represent $17.39 \%$ of the total 46 squamous cell carcinoma.

\section{T Stage}

According to histopathological TNM staging, majority of the tumors were T4 28 (49.1\%) followed by T2 13 (22.8\%) then T1 9 (15.8\%) and T3 7 (12.3\%) (Table 1). Six (66.66\%) of the nine patients who developed positive level $\mathrm{IIb}$ lymph nodes had T4 tumor ( $p=0.217), 2$ had T3 ( $p=0.304)$ and 1 had T2 ( $\mathrm{p}=0.335)$. From the other perspective, $6(21.4 \%)$ of the total 28 T4 tumors had level IIb lymph node metastasis.

\section{Histopathological N Stage}

In histopathological $\mathrm{N}$ classification, 30 (52.6\%) patients had N0, 13 (22.8\%) had N1, 10 (17.5\%) had N2b, 3 (5.3\%) had N2a and only one (1.8\%) with N2c (Table 1$)$. In relation to the nine positive level IIb lymph nodes, 5 (55.6\%) were $\mathrm{N} 2 \mathrm{~b}, 2$ (22.2\%) were N1 and 2 (22.2\%) were N2a. There was statistical significant association between level IIb lymph nodes metastasis and N2b $(p=0.005)$.

\section{Clinical N Stage}

Clinically, out of the 84 neck dissections, 56 necks (66.67\%) were N0 and 28 (33.33\%) were N+. Seven (77.8\%) out of the nine positive level IIb lymph nodes were found in the clinically classified $\mathrm{N}+$ necks ( $p=0.005)$, while $2(22.2 \%)$ of them were found in the clinically classified N0 neck nodes (Graph 1). On the other way, 7 (25\%) out of the total $28 \mathrm{~N}+$ necks were associated with level $\mathrm{IIb}$ nodes metastasis, whereas 2 (3.57\%) out of the total 56 N0 necks had level IIb node metastasis.

\section{Level Ila}

Level IIa lymph node metastasis was documented in $22(26.2 \%)$ neck dissections and all the nine (40.9\%) positive IIb lymph nodes were among them (Graph 2). There was an obvious statistical significant association between metastasis to both levels ( $\mathrm{p}<0.01$ ).

\section{DISCUSSION}

Results from our study showed high incidence (66.66\%) of level IIb node involvement when the primary site of the tumor was in the oral cavity. Considering the total number oral cavity cancers 43, 6 (13.95\%) had level IIb lymph node metastasis. Also, more involvement was documented when the histopathology showed
SCC. The 46 patients having SCC, 17.39\% level IIb nodes resulted positive. Nodal metastasis to level $\mathrm{IIb}$ occurred in 6 (21.4\%) of the 28 patients who had T4 tumors. It was $66.66 \%$ of the nine involved nodal metastasis. There was significant statistical associations between level IIb nodes metastasis and both advanced pathological $\mathrm{N}$ stage and clinically positive lymph nodes. All the patients (100\%) with level IIb positive for metastasis presented metastatic disease in level IIa lymph nodes. Out of the 22 level IIa involvement, $40.9 \%$ had simultaneous level IIb nodes involvement. Considering the total number of neck dissections, the incidence of metastasis to level IIb lymph nodes was $10.7 \%$ which cannot be labeled as negligible.

Involvement of the cervical lymph nodes by metastasis from head and neck tumors poses a serious obstacle to patients survival even when the primary cancer was treated successfully. ${ }^{10}$ Sometimes cervical lymph node metastasis can be found, although the primary tumor is not large and not even progressive. ${ }^{11}$ Metastasis to neck lymph nodes (especially levels I, II and III) from oral cavity primary tumors demonstrates almost the same pattern of

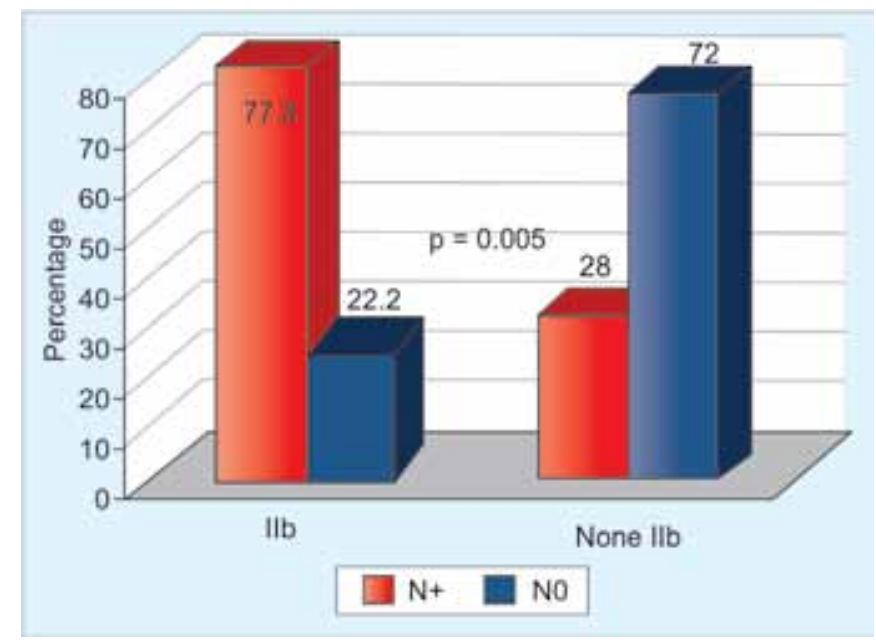

Graph 1: Relationship between level Ilb and clinical neck nodes

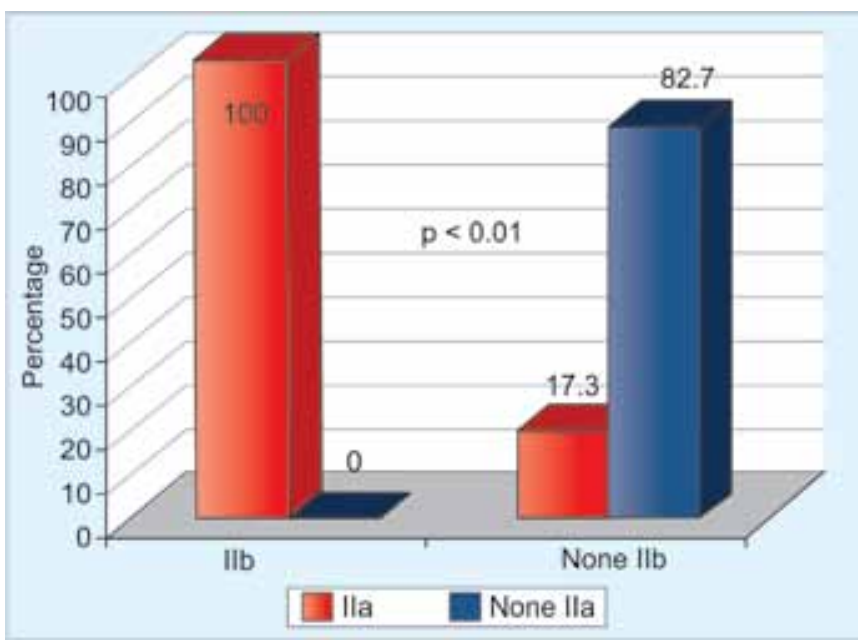

Graph 2: Correlation of level lla metastasis with level Ilb metastasis 
metastasis. ${ }^{12}$ Keeping in mind the postoperative shoulder dysfunction and quality of life ${ }^{9}$ and the incidence of level IIb metastasis from head and neck tumors, is it worth to preserve this level during neck dissection or not?

In our study, the incidence of level IIb metastasis was $3.57 \%$ for clinically N0 necks which is almost close to what was observed in literature, while it is bit higher $(25 \%)$ than what was reported for clinically $\mathrm{N}+$ which may influence the surgeons to think thoroughly to dissect this level after considering the advantages and disadvantages after removing it. A prospective study of 90 SND showed the incidence of level IIb lymph nodes metastasis for clinical $\mathrm{N}+11.1 \%$, for N0 $1.6 \%$ and for all $4.4 \%{ }^{4}$ A meta-analysis study that screened 729 abstracts and 177 fulltext papers about SCC of oral cavity, 332 patients of only 9 papers were included in that analysis. Twenty (6\%) patients were positive for level IIb lymph node metastasis. Three of them $(3 / 20)$ were isolated and $(17 / 20)$ were accompanied by other positive neck levels lymph nodes. ${ }^{13} \mathrm{In}$ Kim et al, ${ }_{1}^{14}$ out of 93 neck dissections of hypopharyngeal SCC, level IIb nodal metastasis was significant in both clinically $\mathrm{N}+$ and $\mathrm{N} 0$ necks, but much more with $\mathrm{N}+$ cases than N0 ( $p=0.007, \mathrm{p}=0.01$, respectively). Alessandra et $\mathrm{al}^{10} \mathrm{had}$ reviewed different prospective studies of clinically N0 necks of 211 patients with laryngeal carcinoma and showed only 3 patients to be level IIb lymph nodes positive. Roberto et $\mathrm{al}^{8}$ study of 114 patients with 148 neck dissections of head and neck cancers, the incidence of level IIb lymph nodes metastasis was 3.3\% for total, $2 \%$ for clinically N0 and $5 \%$ for $\mathrm{N}+$ necks. There was no significant association between clinical $\mathrm{N}$ classification and metastasis to level IIb lymph node $(\mathrm{p}=0.06)$.

In our study, all the $9(100 \%)$ positive level IIb lymph nodes are level IIa lymph node positive for metastasis $(p=0.01)$. Our study showed significant association $(p=0.005)$ between level IIb lymph node involvement and advanced pathological $\mathrm{N}$ staging, i.e. out of the nine positive IIb lymph node in our study, five were $\mathrm{N} 2 \mathrm{~b}(\mathrm{p}=$ 0.005 ). All these results are almost similar to what was reported in literature. Level IIa were found to be positive for lymph nodes metastasis for all patients of positive level IIb. ${ }^{8}$ In Talmi et al, ${ }^{15}$ four out of 102 neck dissections had level IIb lymph nodes metastasis. All of the four neck dissections had level IIa lymph node metastasis and were advanced pathological N staging. Similar study showed statistically significant associations between level IIb and IIa nodes cancerous involvement and between level IIb lymph nodes and advanced pathological $\mathrm{N}$ staging. ${ }^{4}$

Level IIb metastasis is not significantly associated with primary tumor site. ${ }^{4}$ Regarding the association between level IIb and type of the primary tumor, it was shown in the study not to be statistically significant. In the other hand, Elsheikh et al ${ }^{6}$ prefers to preserve level IIb in SND in oral cancer except if the tongue is the primary site of the cancer.

Recognition of risk factors like pathological TNM staging and positive level IIa lymph nodes can help in making a decision whether to dissect level IIb lymph nodes or not. That is why obtaining frozen section biopsy intraoperatively is highly recommended to identify these factors. ${ }^{4}$ Other study ${ }^{8}$ found it to be inaccurate to perform frozen section biopsy to identify level IIa lymph nodes occult metastasis. One study ${ }^{14}$ suggests removing this level in clinically $\mathrm{N}+$ necks, but mostly avoided in clinical N0. While other study recommend to do postoperative radiotherapy to handle level IIb occult micrometastasis in clinically $\mathrm{N}+$ and to dissect it in N0, especially if no radiotherapy was done. ${ }^{15}$

\section{CONCLUSION}

Our opinion about neck dissection for head and neck cancers is that level IIb lymph nodes dissection should be performed in oral cavity cancers also in SCC irrespective of the site and regardless of the $\mathrm{T}$ or $\mathrm{N}$ stage of the tumor. We also recommend to dissect level IIb in any $\mathrm{T} 4$ tumor or N+ necks suggested clinically or diagnosed radiologically. Also, if the level IIa lymph node is suspicious intraoperatively, a frozen section biopsy should be sent for histopathology. If it showed metastasis, both levels IIa and IIb nodes should be dissected. This will decrease the chance of missing level IIb nodes micrometastases. Consequently, incidence of tumor recurrence and distant metastasis will be markedly reduced. A limitation of our study is that we did not investigate the relation between level IIb metastasis and the different sites of oral cavity tumors especially the tongue. More researches are needed in these aspects.

\section{REFERENCES}

1. Mehanna H, Paleri V, West CM, Nutting C. Head and neck cancer-Part 1: Epidemiology, presentation, and prevention. BMJ 2010;341:c4684.

2. Roepman P, de Jager A, Groot Koerkamp MJ, Kummer JA, Slootweg PJ, Holstege FC. Maintenance of head and neck tumor gene expression profiles upon lymph node metastasis. Cancer Res 2006;66(23):11110-11114.

3. Robbins KT, Clayman G, Levine PA, et al. Neck dissection classification update: revisions proposed by the American Head and Neck Society and the American Academy of Otolaryngology, Head and Neck Surgery. Arch Otolaryngol, Head and Neck Surg 2002;128(7):751-758.

4. Silverman DA, El-Hajj M, StromeS, Esclamado RM. Prevalence of nodal metastases in the submuscular recess (level IIb) during selective neck dissection. Arch Otolaryngol, Head and Neck Surg 2003;129(7):724-728. 
5. Villaret AB, Piazza C, Peretti G, Calabrese L, Ansarin M, Chiesa F, et al. Multicentric prospective study on the prevalence sublevel IIb metastases in head and neck cancer. Arch Otolaryngo, Head and Neck Surg 2007;133(9):897-903.

6. Elsheikh MN, Mahfouz ME, Elsheikh E. Level IIb lymph nodes metastasis in elective supraomohyoid neck dissection for oral cavity squamous cell carcinoma: a molecular-based study. Laryngoscope 2005;115(9):1636-1640.

7. Lee SY, Lim YC, Song MH, Lee JS, Koo BS, Choi EC. Level IIb lymph node metastasis in elective neck dissection of oropharyngeal squamous cell carcinoma. Oral Oncol 2006;42(10):1017-1021.

8. Santoro R, Franchi A, GalloO, BuraliG, de' Campora E. Nodalmetastases at level IIb during neck dissection for head and neck cancer: clinical and pathologic evaluation. Head Neck 2008;30(11): 1483-1487.

9. Kraus DH, Rosenberg DB, Davidson BJ et al. Supraspinal accessory lymph node metastases in supraomohyoid neck dissection. Am J Surg 1996;172(6):646-649.

10. Rinaldo A, Elsheikh MN, Ferlito A, et al. Prospective studies of neck dissection specimens support preservation of sublevel
IIB for laryngeal squamous carcinoma with clinically negative neck. J Am Col Surg 2006;202(6):967-970.

11. Capote A, Escorial V, Munoz-Guerra MF, Rodriguez-Campo FJ, Gamallo C, Naval L. Elective neck dissection in early-stage oral squamous cell carcinoma-does it influence recurrence and survival? Head and Neck 2007;29(1):3-11.

12. Shah JP, Candela FC, Poddar AK. The patterns of cervical lymph node metastases from squamous carcinoma of the oral cavity. Cancer 1990;66(1):109-113.

13. Lea J, Bachar G, Sawka AM, et al, Goldstein DP. Metastases to level IIb in squamous cell carcinoma of the oral cavity: a systematic review and meta-analysis. Head Neck 2010;32(2): 184-190.

14. Kim YH, Koo BS, Lim YC, Lee JS, Kim SH, Choi EC. Lymphatic metastases to level IIb in hypopharyngeal squamous cell carcinoma. Arch Otolaryngol, Head Neck Surgery 2006;132(10): 1060-1064.

15. Talmi YP, Hoffman HT, Horowitz Z, et al. Patterns of metastases to the upper jugular lymph nodes (the submuscular recess). Head Neck 1998;20(8):682-686. 